

\section{REVISTA \\ TEORÍA Y PRÁCTICA \\ DE LA \\ ARQUEOLOGÍA HISTÓRICA LATINOAMERICANA}

ISSN: 2250-866X (impreso) | ISSN: 2591-2801 (en línea)

AÑO VIII, VOLUMEN 9, PRIMAVERA DE 2019

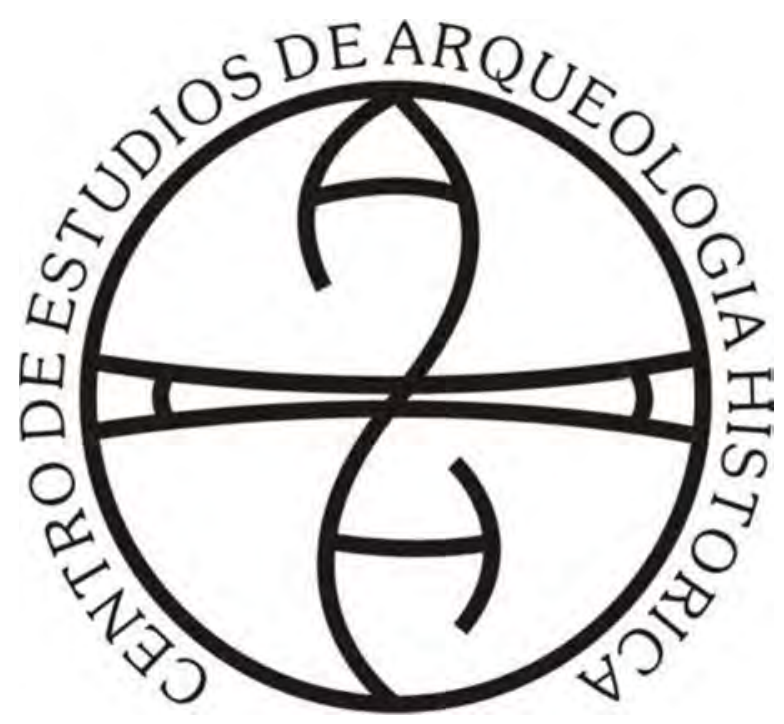

CENTRO de Estudios de ARqueOlOGía HistóricA

FACULTAD DE HUMANIDADES Y ARTES | UNIVERSIDAD NACIONAL DE ROSARIO 
(Universidad Nacional de Rosario, Universidad Nacional de Río Cuarto,

Universidad Nacional de San Juan, Universidad de la República, Universidad Nacional de Trujillo)

\author{
AUTORIDADES DE LA UNIVERSIDAD NACIONAL DE ROSARIO \\ RECTOR: Lic. Franco Bartolacci \\ VICE-RECTOR: Od. Darío Macía \\ SECRETARIO GENERAL: Prof. José Goity \\ SECRETARIA ACADÉMICO Y DE APRENDIZAJE: Dr. Marcelo Vedrovnik \\ SECRETARÍA DE CIENCIA TECNOLOGÍA E INNOVACIÓN \\ PARA EL DESARROLLO: Ing. Guillermo Montero.
}

\author{
AUTORIDADES DE LA FACULTAD DE HUMANIDADES Y ARTES \\ DECANO: Prof. Alejandro Vila \\ VICEDECANA: Prof. Marta Varela \\ SECRETARIA ACADÉMICA: Dra. Marcela Coria
}

\author{
AUTORIDADES DEL CENTRO DE ARQUEOLOGÍA HISTÓRICA \\ DIRECTORA: Dra. Ana Rocchietti \\ SECRETARIA: Prof. Nélida de Grandis \\ PROSECRETARIA: Lic. Marianela Bizcaldi
}

DIRECTORAS - EDITORAS:

Dra. Ana Rocchietti y Prof. Nélida De Grandis

SECRETARIA DE EDICIÓN: Dra. Irene Dosztal

Este número es co-edición de las ponencias

del VIII Congreso Nacional de Arqueología Histórica (2018) entre:

Centro de Estudios en Arqueología Histórica: Directora Ana Rocchietti

Centro de Estudios en Arqueología Regional: Director Fernando Oliva

Centro de Estudios en Arqueología Subacuática: Directora Mónica Valentini

Departamento de Arqueología, Escuela de Antropología: Director Fernando Oliva
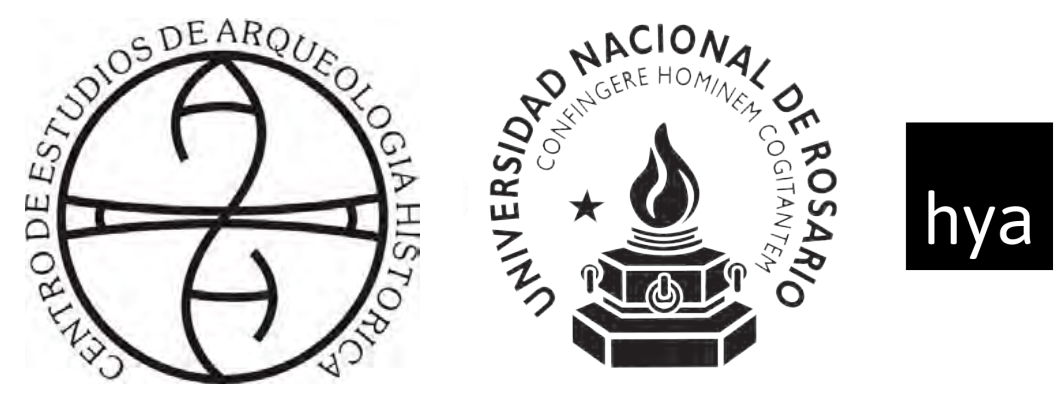

Facultad de Humanidades

y Artes_UNR
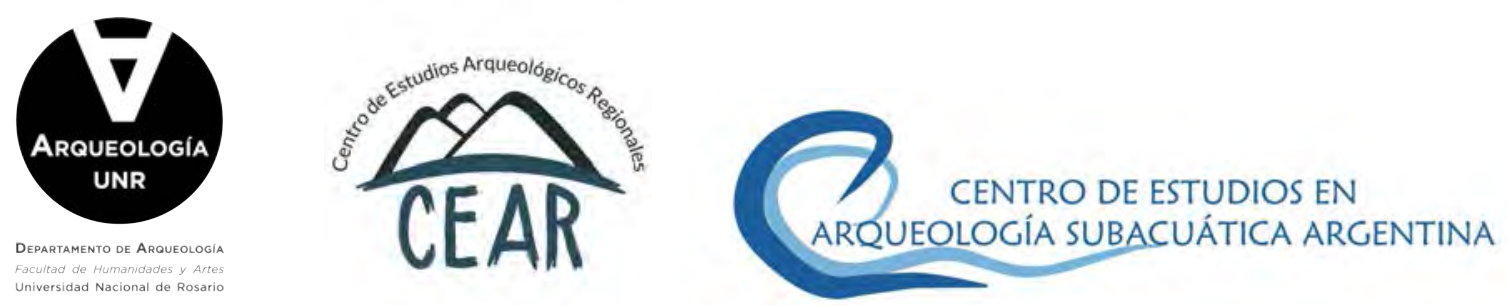
Comité Permanente de los Congresos Nacionales de Arqueología Histórica

Dr. Daniel Schávelzon (Universidad Nacional de

Buenos Aires)

Prof. María Teresa Carrara (Universidad Nacional de Rosario)

Prof. Carlos Baldassarre (Museo Municipal de Río

Grande, Tierra del Fuego) in memoriam

Dr. Mariano Ramos (Universidad Nacional de Luján, CONICET)

Dr. Horacio Chiavazza (Universidad Nacional de Cuyo)

Dra. Ana María Rocchietti (Universidad Nacional de

Rosario, Universidad Nacional de Río Cuarto)

Lic. Facundo Gómez Romero (Universidad Autónoma

de Barcelona)

\section{Comité Científico}

Dra. Tânia Andrade Lima (Universidade Federal do Rio de Janeiro)

Prof. Réginald Auger (CELAT/Département des

Sciences Historiques, Université Laval, Canadá)

Dr. Roberto Bárcena (Universidad Nacional de Cuyo, CONICET)

Dra. Marta Bonaudo (Universidad Nacional de Rosario, CONICET)

Dr. Leonel Cabrera (Universidad de la República, Uruguay)

Dr. Luis María Calvo (Universidad Católica de Santa

$\mathrm{Fe})$

Prof. Juan Castañeda Murga (Universidad Nacional de

Trujillo, Perú)

Dr. Carlos Ceruti (Museo de Ciencias Naturales y

Antropología "Prof. Antonio Serrano”. Paraná)

Dr. Horacio Chiavazza (Universidad Nacional de Cuyo)

Dra. Silvia Cornero (Universidad Nacional de Rosario)

Prof. Pedro Paulo Funari (Universidade Estadual de

Campinas, Brasil)

Lic. Jorge A. Gamboa Velásquez (Universidad Nacional

Santiago Antuñez de Mayolo, Perú)

Dr. Eduardo Alejandro García (Universidad Nacional de

San Juan, CONICET)

Prof. Nélida De Grandis (Universidad Nacional de

Rosario)

Dr. Juan Bautista Leoni (Universidad Nacional de

Rosario, CONICET)

Dra. Amancay Martínez (Universidad Nacional de San

Luis)

Dra. Catalina Teresa Michieli (Universidad Nacional de

San Juan, CONICET)

Lic. Fernando Oliva (Universidad Nacional de Rosario)
Ing. Adrián Pifferetti (Universidad Tecnológica Nacional Regional Rosario)

Dr. Mariano Ramos (Universidad Nacional de Luján, CONICET)

Dra. Ana María Rocchietti (Universidad Nacional de Rosario)

Dr. Daniel Schávelzon, (Universidad Nacional de Buenos Aires, CONICET)

Dra. Carlota Sempé (Universidad Nacional de La Plata)

Dr. Mario Silveira (Universidad Nacional de Buenos

Aires)

Dra. Silvia Simonassi (Universidad Nacional de Rosario)

Dra. Alicia Tapia (Universidad Nacional de Buenos Aires, Universidad Nacional de Luján)

Lic. Mónica P. Valentini (Universidad Nacional de Rosario)

Agrim. Benito Vicioso (Universidad Nacional de Rosario)

\section{Evaluaron este volumen}

Roberto Bárcena, María Teresa Boschin, Leonel Cabrera, Ulises Camino, María Rosa Carbonari, Carlos Ceruti, Horacio Chiavazza, Nicolás Ciarlo, Silvia Cornero, Eduardo Crivelli, Javier García Cano, Martín Gentinetta, María Laura Gili, Carlos Landa, Matilde Lanza, Melina Malandrino, Sebastián Pastor, Victoria Pedrotta, Josefina Piana, Mercedes Podestá, Mariano Ramos, Daniel Schavelzon, Diana Tamburini, Mónica Therrien, Mónica Valentini y María Teresa Boschin

\section{Diseño y diagramación}

Eugenia Reboiro

(eugenia.reboiro@gmail.com)

\section{Curadoría}

Ana Rocchietti e Irene Dosztal

Foto de tapa: Material arqueológico, del texto de Tapia et al.

\section{Propietario responsable:}

Facultad de Humanidades y Artes, Universidad Nacional de Rosario Centro de Estudios de Arqueología Histórica

Entre Ríos 758. Rosario, provincia de Santa

Fe (2000). Argentina.

Telf.: +54 (0341) 4802670

E-mail: ceahunr@gmail.com

Decreto Ley 6422/57 de Publicaciones

Periódicas 


\section{Índice}

Editorial

El origen del patrimonio histórico y su conservación integral en la contemporaneidad.

Yanina Aguilar

Prospección de basurales históricos de la ciudad de Rosario.

Soccorso Volpe y Gustavo Fernetti

Desconstrucción de un paisaje urbano. El Montevideo del extramuros colonial, aportes de la arqueología a su identidad actual. Ana Gamas

La Calota de Ameghino: reconsiderando un viejo tema desde la arqueología histórica.

Daniel Schávelzon

Análisis zooarqueológico de los restos hallados en una estructura sanitaria vinculada a momentos tempranos del actual barrio de Belgrano .71 Mario Silveira, Horacio Padula, Ricardo Orsini y Eva Bernat

La medida del tiempo: el reloj solar jesuita de La Cruz, provincia de Corrientes.

Fernando Oliva y María Cecilia Panizza

Sitio La Quinta: arqueología rural de campos serranos.

Flavio Ribero

Si no hay tabaco que no se note. Prácticas fumatorias en el fortin La Perra (1883-1885), La Pampa.

Alicia Tapia, Virginia Pineau y Melisa Ayelén Auge

El uso del tabaco y las pipas de caolin (clay pipes) en la frontera sur y oeste de la provincia de Buenos Aires en el siglo XIX.. .127

María del Carmen Langiano y Julio Fabián Merlo 
Patrimonio natural, urbano y arquitectónico de la costa en Mar del Plata. Federico Negroni

Huellas urbanas.

Ezequiel Serrot

Paisaje y patrimonio. La industria taninera en el siglo XX.

Provincia de Santa Fe.

Cristina Pasquali, Paola Milicic y Lara Ferré

Los desafíos de las arqueologías históricas latinoamericanas.

Andrés Zarankin

Sellos entre escombrales. Las lozas en el registro urbano

del Área Fundacional de Mendoza (siglo XIX y principios del siglo XX)

Lorena Puebla y Horacio Chiavazza

Evolución edilicia de la Plaza de Mayo de la Ciudad

de Buenos Aires (1580-1853).

Nicolás Ferrino

Gestión patrimonial en el rescate arqueológico y futuro Museo de Sitio

en Moreno 550, Ciudad Autónoma de Buenos Aires, Argentina.

María Eva Bernat, Ricardo Orsini, Horacio Padula y Mario Silvera 


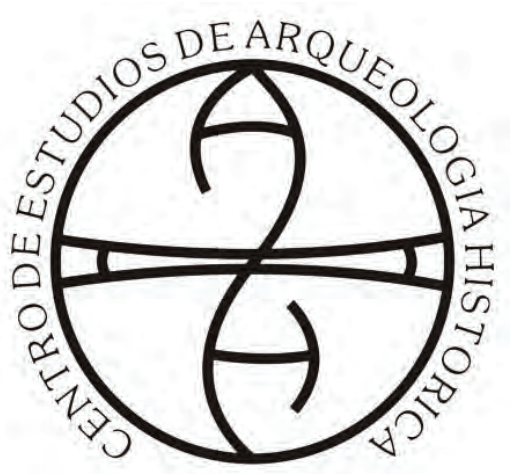

Centro de Estudios de Arqueología Histórica

Universidad Nacional de Rosario
Teoría y Práctica de la Arqueología Histórica

Latinoamericana | Año VIII. Vol. 9 | 2019

Revista del Centro de Estudios de Arqueología

Histórica, Facultad de Humanidades y Artes, Universidad Nacional de Rosario

https://teoriaypracticaah.unr.edu.ar/index.php/index ceahunr@gmail.com

ISSN en línea: 2591-2801

ISSN versión impresa: 2250-866X

\title{
Prospección de basurales históricos DE LA CIUDAD DE ROSARIO
}

\author{
Socorso Volpe* y Gustavo Fernetti**
}

\begin{abstract}
Resumen
Los basurales suelen ser una forma privilegiada de acceso a la historia de una ciudad. La población de Rosario, una urbe que creció desde mediados de la década de 1850 y sobre todo a partir del auge migratorio entre 1880 y 1920, produjo numerosos basurales de diverso tipo. La introducción de mercancía industrial importada pobló los basurales rosarinos de innumerables fragmentos de composición heterogénea, pero que permiten -120 años después- aproximarse a la ciudad en formación.

Para realizar estos potenciales estudios, durante los años 2014 a 2017 se relevaron en la ciudad más de 100 basurales de todo tipo siguiendo una metodología específica para ello.

El trabajo realizado servirá para futuras investigaciones, intentando dotar de material referenciado un panorama complejo y variado, pero útil para la exploración arqueológica del pasado rosarino.

Palabras clave: Arqueología urbana, basurales, Rosario
\end{abstract}

\begin{abstract}
Usually, landfills are a privileged way of accessing the history of a city. The folk of Rosario, a city that grew from the mid-1850s and especially from the migration boom between 1880 and 1920, produced numerous dumps of various kinds. The introduction of imported industrial merchandise, filled the Rosario garbage dumps of innumerable fragments of heterogeneous composition, but that allows - 120 years later- to approach the city in formation.

To carry out these potential studies, during the years 2014 to 2017, more than 100 landfills of all types were surveyed, following a specific methodology for this.

The work carried out will be useful for future research, trying to provide referenced material with a com-

* Centro de Estudios en Arqueología Histórica, Facultad de Humanidades y Artes, Universidad Nacional de Rosario. ninosoccorso@yahoo.com.ar

** Centro de Estudios en Arqueología Histórica, Facultad de Humanidades y Artes, Universidad Nacional de Rosario. arqfernetti@hotmail.com
\end{abstract}


plex and varied panorama, but useful for the archaeological exploration of Rosario’s past.

Keywords: Urban archaelogy, landfills, Rosario

\section{Introducción}

Durante la introducción del capitalismo luego del ascenso del presidente Mitre, y sobre todo con la llegada masiva de capitales y migración europea después de 1880, Rosario fue una plaza comercial activa, con alto intercambio de bienes pecuarios “del país” y productos manufacturados europeos.

La concurrencia de mercancías industriales que Argentina era incapaz de fabricar respondía al consumo realizado por la gran cantidad de población resultado del proceso inmigratorio iniciado en la década de 1870, del cual Rosario fue también receptor. A la vez, el salto demográfico resultado del proceso (casi triplicando en 1915 la población de 1870) propició la aparición de nuevas áreas edificadas, establecimientos e instituciones insólitos en cantidad y tipo y la complejización de las relaciones sociales.

El deseo de residencia de los nuevos contingentes, consolidó el proceso de urbanización mencionado y de la ciudad rural de principios del siglo XIX, Rosario pasó a ser una ciudad aún "dispersa” (Galimberti, 2016, p.70) pero en formación, esta urbanización afectó fuertemente la condición de la población al crecer, por la actividad comercial, según el sentido centro -norte / centro-sur y centro-oeste. Mientras que el primer sentido o eje industrial formó los barrios obreros Refinería, Talleres e Industrial, el segundo eje de infraestructura fue formado por el Matadero y el puerto definió el barrio de la Quema y Tablada (Falcon, 2005, p.44).

Separadas de estas urbanizaciones primigenias y fuertemente dependientes de la actividad económica, aparecieron dos urbanizaciones de recreo para las élites: el Pueblo Alberdi en 1776 hacia el norte y la urbanización Nueva Corina hacia el sur. La separación de las actividades económicas de la Refinería y los Talleres era suficiente para establecer una diferenciación al punto que Pueblo Alberdi era políticamente autónomo. Nueva Corina, en el otro extremo, pertenecía a Rosario y se comunicaba, hacia 1905, mediante una línea de tramway.

Hacia 1920, ya atenuado el flujo de la inmigración masiva, Rosario podía verse como una ciudad urbanísticamente dinámica, en transformación constante, dependiente del intercambio comercial agrícola mediante su puerto.

Los comienzos de la sustitución de importaciones, iniciada con la Primera Guerra Mundial, concluyó en un proceso de industrialización que colocó en los comercios gran cantidad de mercancía local, a la vez que empezaron a introducirse en el mercado bienes de consumo estadounidenses, reemplazando cada vez más los productos ingleses y franceses.

Ya en la década de 1940 y sobre todo con el peronismo en el gobierno (1945-55) la industrialización se convirtió en una fuerte política del estado, que pervivió hasta la década de 1970 (Mugica y Martin 2001, p.180).

\section{Basurales y arqueología}

El basural puede considerarse una evidencia de consumos, en tanto es el recolector de fragmentos de mercancías de uso y consumibles, por ello la basura es heterogénea y a la vez su deposición no siempre es sistemática: la dinámica física de los basurales depende de numerosas variables en la deposición, como el sistema de recolección y volcado, el tratamiento posterior de la basura, el reuso y los traslados posdeposicionales. La basura puede ser descartada, pero luego seleccionada, procesada o trasladada (Schiffer, 1990, p.92). 
Teoría y Práctica de la Arqueología Histórica Latinoamericana | Año VIII. Vol. 9 | 2019

ISSN en línea: 2591-2801 | ISSN versión impresa: 2250-866X

Esto no quiere decir que no hubiese, en la formación y transformación del basural, una “lógica deposicional”, pero esa lógica no es una ley general o una dinámica regular y extensible a todos los casos que podrían analizarse.

A la vez, el basural es un indicador del proceso de urbanización: los basurales se ubicarían en sitios específicos, socialmente definidos y no aleatorios (Fernetti, 2015, p.12). Estas ubicaciones dependerían de los espacios y su relación con las poblaciones, la consideración del espacio de deposición y las condiciones socioeconómicas de la sociedad que generó el basural. La actividad que lo ha generado es también una condicionante en la formación. Los basurales técnicos, por ejemplo, dependen de una actividad determinada. Los talleres de fundición de metales o los hospitales generan basuras específicas, pero también heterogéneas en tanto pueden combinar los descartes propios de la actividad específica -para los ejemplos escorias o restos de medicamentos- con otros, producto del consumo de las personas que desarrollan esas actividades, que pueden ser comunes a otros grupos o poblaciones sin esas acciones particulares.

En ese marco, los basurales también revelan la inserción de una sociedad que cambia en una economía emergente (Colasurdo y Sartori, 2011, p.141; Colasurdo, 2012, p. 277). Los bienes cuyos fragmentos se recuperan implican un modo de entender su adquisición. Las frecuencias de los fragmentos (cantidades) pero también su recurrencia (qué tipo de fragmento aparece) y su concurrencia (su aparición en otros basurales) permiten indagar sobre la compra y uso de los objetos originales y compararlos con los hallados en otros sitios.

Por ejemplo, la eventual alta frecuencia de costosa porcelana europea de calidad en un basural, implicaría -comparativamente- una diferencia social respecto a otro, pero debe ser contrastado con otras concurrencias de ese material para otros sitios. Y también es necesario un contraste con la aparición de otros bienes de consumo específico y con la documentación histórica o etnográfica disponible.

Así, el múltiple entrecruzamiento de datos permite considerar a los basurales no ya como una deposición física y materialmente aleatoria de fragmentos heterogéneos, sino como resultado de un ordenamiento social y económico.

Los basurales, de este modo, son espacios/paisajes cuyo análisis permite acceder a los consumos y actividades de los grupos que descartaron los fragmentos de bienes comerciales o consumibles dentro una “domesticidad” (López Lillo, 2016, p.337).

Pero dado que también es un indicador de los modos de urbanización y de inserción en un modo de producción, la detección de los basurales y su posterior análisis implica analizar en forma indirecta la sociedad y la ciudad como un site-city ${ }^{1}$ (Cressey y Stephens, 1982, p.44). resultado de una dinámica socioeconómica que excede el acto cotidiano de deposición de basuras, pero que lo incluye.

Su localización, cualificación y encuadre cronológico (aunque provisional) permitirían así establecer áreas arqueológicas específicas, en la inteligencia que éstas indicaron el modo en que la ciudad fue construida desde el siglo XVIII hasta hoy.

Al mismo tiempo el análisis de la producción y el consumo, de este modo, permitiría acceder tanto a las dinámicas sociales inmediatamente derivadas de los fragmentos -que se producía y qué consumíacomo a las que se derivan de análisis secundarios, o sea la comparación entre sitios y por ende, de las poblaciones de esa ciudad "dispersa” pero en consolidación.

Estos basurales fueron resultado de la construcción socioeconómica de la ciudad y su identificación permitiría el análisis de la historia rosarina desde la perspectiva de la arqueología urbana.

El objetivo del presente trabajo fue informar de la detección y relevamiento esos basurales históricos de Rosario, encuadrados cronológicamente entre fines del siglo XVIII y principios del siglo XX. Las 
tareas de relevamiento se realizaron entre 2014 y 2016, llegándose a localizar 127 basurales de distintas escala urbana y riqueza en fragmentos.

\section{El concepto de prospección empleado}

La Ley Nacional $25.743^{2}$ intenta proteger los sitios arqueológicos y paleontológicos, mediante tres acciones fundamentales y concomitantes: otorgar concesiones para la investigación de sitios arqueológicos (Figura 1), obligar al registro de los fragmentos hallados y reprimir a las personas que actúen sobre los sitios sin concesión autorizada.
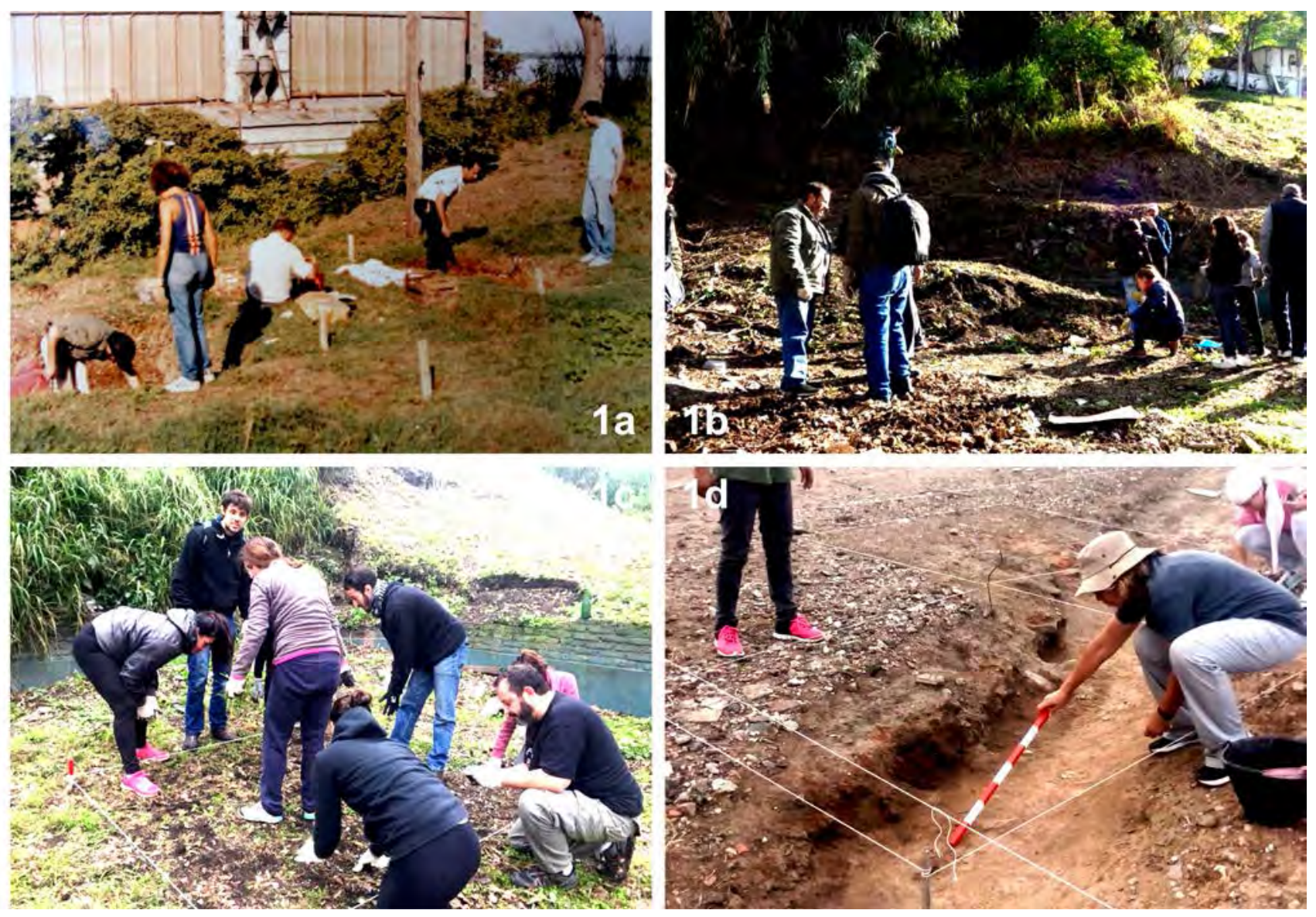

Figura 1- Trabajos arqueológicos autorizados y realizados en Rosario: 1a- Trabajos de excavación sistemática en el sitio MCU1 “La Basurita”, c. 1990. 1b- Prospecciones sistemáticas, 2019. 1c y 1dExcavaciones sistemáticas de 2017-19.

En su artículo primero, se establece como objetivo "Es objeto de la presente ley la preservación, protección y tutela del Patrimonio Arqueológico y Paleontológico como parte integrante del Patrimonio Cultural de la Nación y el aprovechamiento científico y cultural del mismo. En su artículo 39, la ley enuncia: 
Teoría y Práctica de la Arqueología Histórica Latinoamericana | Año VIII. Vol. 9 | 2019

ISSN en línea: 2591-2801 | ISSN versión impresa: 2250-866X

Las personas que realicen por sí, u ordenaren realizar a terceros, tareas de prospección, remoción o excavación en yacimientos arqueológicos y paleontológicos sin solicitar la correspondiente concesión ante la autoridad competente, serán pasibles de multa, la que se fijará de acuerdo a la magnitud de la alteración realizada y el decomiso de todos los objetos de naturaleza arqueológica o paleontológica que hayan sido reunidos, aunque se encuentren en posesión de terceros que aleguen adquisición de buena fe (art. 31).

Este artículo presentaría una severa contradicción lógica.

El concepto de “yacimiento arqueológico (y paleontológico)” debería ser establecido antes de poder reprimir su prospección. La ley en su artículo consideró al yacimiento como preexistente a lo que se reprime.

Se podría preguntarse pues cómo se definiría in situ un yacimiento sin realizar las mínimas prospecciones. Por lo tanto, la prospección, al estar interdicta por ley, no permitiría la identificación de un yacimiento.

Esto llevaría a otra contradicción. Si el yacimiento (la ley no habla de sitio) fuese arqueológico ¿Qué tipo de materiales lo constituirían como tal? Nuevamente, es la observación sistemática y la eventual recolección la que definiría el carácter arqueológico, puesto que no cualquier deposición sería antigua y mucho menos arqueológica. Si el carácter de yacimiento fue definido con antelación a su detección y cualificación como tal, también su carácter arqueológico sería previo a cualquier observación.

Paradójicamente también, la Ley Nacional 25.743, al promulgarse tuvo como misión: Crear y organizar el Registro Nacional de Yacimientos, Colecciones y Objetos Arqueológicos y el Registro Nacional de Yacimientos, Colecciones y Restos Paleontológicos, con la información que se requerirá a las jurisdicciones locales (Art.5. a).

Cabría preguntarse pues, cómo crear un registro nacional, basándose en sitios cuya identificación y cualificación arqueológica dependería de una actividad prohibida. Obviamente mediante las excavaciones concesionadas, pero ¿cómo saber dónde excavar?

Así, la prospección que quedó prohibida por ley -bajo sanción- no tuvo técnicamente un mecanismo analítico mínimo para la positiva localización, identificación y cualificación de sitios potenciales, sin por ello contradecir el texto normativo.

¿En qué consistiría esta prospección prohibida por la ley, pero de la cual depende la conformación de un sitio a proteger por ley?

Si bien la ley no establece la mecánica prospectiva, puede suponerse que es aquélla que acarrea la destrucción total o parcial de un sitio potencialmente arqueológico, ya que existen penalidades severas. Frecuentemente la acción de coleccionistas y arqueólogos o historiadores aficionados, personal no cualificado de museos, turistas y curiosos alteraron de manera irreversible los sitios, al retirar evidencia sin registro alguno. Pero la prospección prohibida, por otro lado, no sería necesariamente destructiva.

Podrían enumerarse distintos casos para los basurales rosarinos:

Tipo 1- Basurales no visibles. En un primer grupo de casos, los fragmentos se hallarían invisibles, constituyendo basurales abandonados, ocultos bajo el manto vegetal, pisos, escombros, rellenos o deposiciones más modernas. Para estos casos, la prospección necesariamente implica una intervención material, para al menos confirmar los datos de la documentación histórica disponible, si la hubiese. Ello estaría prohibido por la ley. Sin embargo, no resultaría esencial para estimar la posición del basural la evidencia inmediata de fragmentos en contexto edáfico. La evidencia documental de poblaciones (a 
veces incluso poblaciones numerosas) puede indicar posibles basurales producto de esas poblaciones, aunque como un área general y sin una localización precisa. La prospección mediante cateos, calicatas y trincheras exploratorias no sería del todo necesaria, aunque sí asertiva, pero dependiendo de cada sitio. El basural sería una aproximación y no un sitio.

Tipo 2- Basurales visibles superficialmente formando suelo arqueológico. Para un segundo grupo de sitios, éstos podrían ser definidos sólo por la observación. En arqueología urbana -el tema de este trabajo- la localización de sitios dependería casi siempre de la observación de concentraciones, transformaciones edáficas antrópicas y asociaciones con la arquitectura. La documentación histórica y la evolución de la propiedad inmueble definirían también la potencialidad arqueológica de ciertos espacios de deposición vinculados a áreas documentadas de vivienda o -al menos- habitación humana o animal. En todos estos casos, la "prospección" se reduciría a observaciones preliminares (registro geográfico y topográfico, fotografiado, etcétera) y trabajo de gabinete, definiéndose el sitio por la asociación entre el material disponible y el registro observacional. Los fragmentos visibles podrían ser fotografiados y dejados en el lugar, ya que su georreferencia estaría (hablando en general) muy afectada por la dinámica edáfica y antrópica, dado que se encuentran expuestos ya que, como se dijo, son visibles.
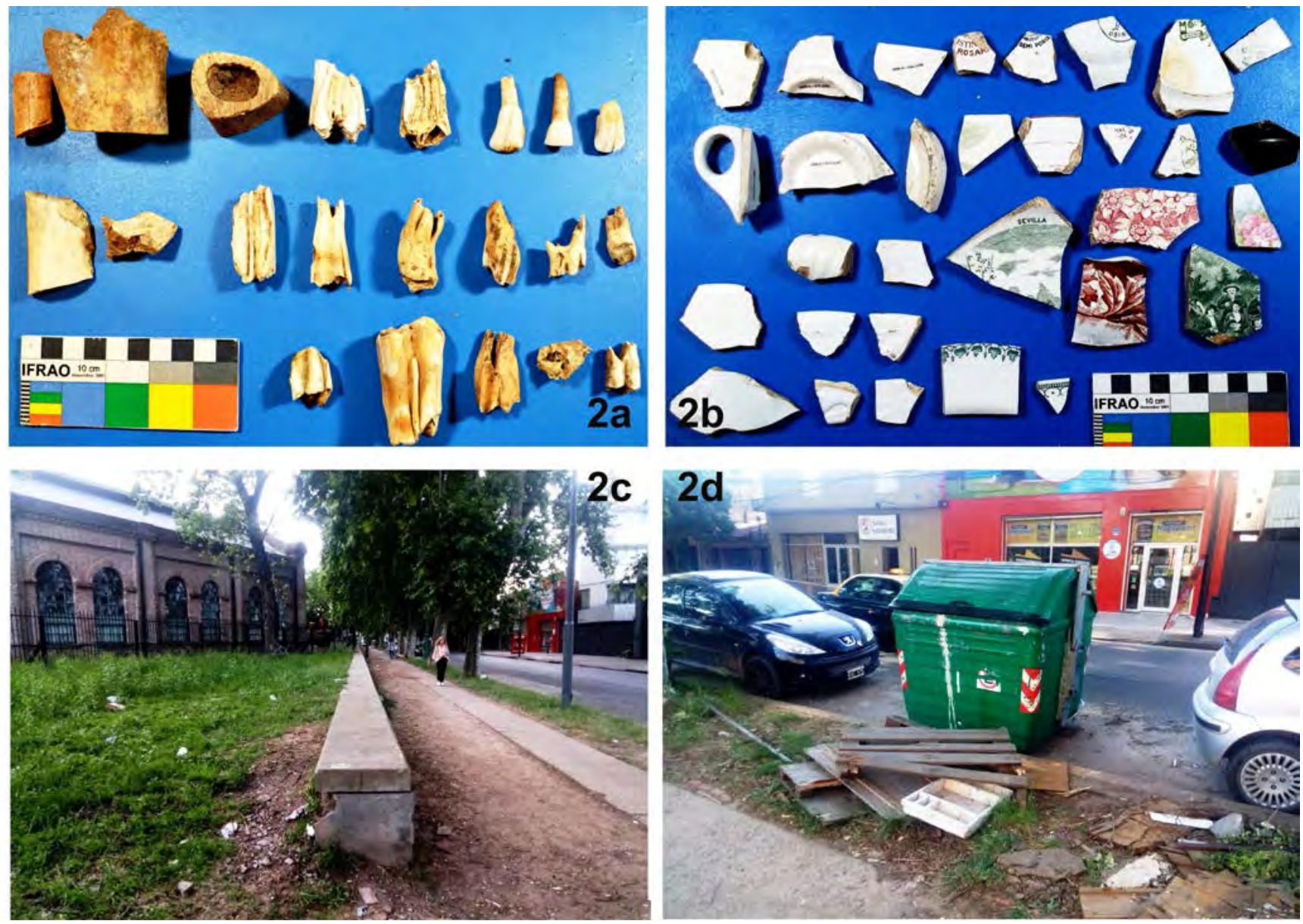

Figura 2-Recolección superficial no destructiva por calle Junín (Sitio JUN3 y JUN5). 2a- Huesos hallados en JUN3, antiguo tambo c. 1905. 2b-Lozas del barrio obrero (JUN5). 2c y 2d-Aspecto de los sitios prospectados JUN6 y JUN7, sobre calle Junín. 
Tipo 3- Basurales visibles en superficie y con alteración en profundidad. En un tercer grupo de casos, la evidencia se ubicó generalmente a plena vista, aunque en sitios alterados constantemente por la dinámica urbana e incluso física del sitio. Las veredas de paredones de fábricas y talleres, los laterales de las vías férreas, los "huecos" históricos, los playones ferroviarios de reserva, constituyeron espacios de deposición de la población de los barrios (Figura 2). A diferencia de las plazas y parques, por lo general vedados al volcado de la basura, estos lugares se utilizaron como un paisaje cultural definido y al aire libre. El resultado consiste fragmentos de todo tipo, hoy se observan en espacios de acceso público, donde este material está permanentemente trasladado y afectado por el paso de peatones y el tránsito vehicular. Para este caso, la evidencia es perfectamente observable, pero el sitio no es localizable con certeza, ya que es un espacio difuso ("nube”, “mancha”) del cual ni siquiera podría decirse que existe una concentración nuclear de bordes netos. La prospección en este caso no sería destructiva, por la sencilla razón de que el sitio está ya en mayor o menor medida destruido como espacio de construcción original recuperable. Como técnica de campo, la recolección “registrada” sería casi contradictoria y no habría una referencia fiable, ya que estaría aplicada a fragmentos trasladados por el paso de peatones o vehículos.

Tipo 4- Basurales de escala urbana. Para los grandes basurales, la formación y su estructura serían siempre de escala urbana. Los miles de fragmentos depositados durante un siglo -para Rosario- forman contextos extensos, con áreas de deposición que pueden superar una manzana urbana. La prospección de estos basurales es compleja, puesto que la simple y puntual observación no alcanzaría a definirlos como basurales arqueológicos, sobre todo la continuarse hoy con el hábito de arrojar basura. Por otro lado, cuantitativamente el retiro de material -fragmentos- no alteraría el sitio, ya que sería sumamente aleatorio que los materiales retirados eliminen un estrato, un cono de deyección completo o un componente. Dicha eliminación es el verdadero riesgo del coleccionismo o de la arqueología amateur y que la ley trata de evitar y reprimir en su artículo 38.

Tipo 5- Basurales descubiertos por obras privadas o del estado. En un quinto grupo, las obras de infraestructura modifican también el estado y localización de los fragmentos, alterando todo el sitio. Excavaciones y demoliciones borran los restos de viejos asentamientos y dada la localización pública o semipública de los basurales, eliminan toda evidencia de su existencia. En este caso, el basural apareció generalmente al efectuarse las obras, en los cortes que provocan las máquinas y el accionar de los obreros. La prospección es para estos casos post-facto, se concurre al sitio para rescatar fragmentos que están en proceso de desaparición. Pero también para registrar contextos de deposición, estratificaciones y estructuras hasta ese momento ocultas. Las ordenanzas municipales rosarinas han quedado en la práctica caducas frente a la ley y su organismo de aplicación (el Ministerio de Cultura Provincial). Sin embargo podrían ser aplicadas para la articulación con las obras de ingeniería o arquitectura proyectadas. Estas prospecciones podrían considerarse "monitoreos” ya que dependen de cómo se realizan las obras, pudiéndose detener momentáneamente las mismas para retirar o registrar.

Tipo 6- Demoliciones y ruinas. Si bien no son basurales, las estructuras murarias y sus relictos (escombros) permiten establecer georreferencias asociadas a los basurales, y se consideran deben ser registradas de modo separado, sobre todo porque su planta podría contener un basural doméstico.

Nótese en los casos 4 y 5 que la recolección puede ser efectuada bien por el tamaño del universo de fragmentos (caso 4) o bien por su eventual desaparición (caso 5), pero no es una necesidad conceptual la recolección superficial de fragmentos para formar una muestra representativa (caso 4) o para "rescatar" 
el sitio (caso 5). Para ambos casos, la recolección superficial no alteraría el sitio o bien impediría la destrucción de una evidencia de posición, por parte de las obras públicas o privadas (Volpe 2000).

En síntesis y observando estos casos, se podría decir que la prospección -como excavación- no es una acción necesaria y aplicable a todos los sitios, sino sólo a aquéllos que se hallan ocultos, de los que sólo se dispone evidencia documental o muy poco material en superficie. En el resto, la prospección se reduce a la observación sistemática, en el sentido de un acto formalizado por el carácter profesional de quien prospecta.

La siguiente tabla (Figura 1) resume los sitios prospectados y clasificados por tipo.

\begin{tabular}{|c|c|c|c|}
\hline TIPO & BASURAL & DESCRIPCION & $\begin{array}{l}\text { PROSPECCION } \\
\text { REALIZADA }\end{array}$ \\
\hline 1 & NO VISIBLE & $\begin{array}{l}\text { BASURALES OCULTOS BAIO MANTO } \\
\text { VEGETAL, PISOS, ESCOMBROSO } \\
\text { DEPOSICIONES MODERNAS }\end{array}$ & $\begin{array}{l}\text { OBSERVACIONY } \\
\text { REGISTRO }\end{array}$ \\
\hline 2 & $\begin{array}{c}\text { VISIBLE } \\
\text { SUPERFICIALMENTE }\end{array}$ & $\begin{array}{l}\text { BASURALES CON FRAGMENTOS } \\
\text { FORMANDO SUELO ARQUEOLÓGICO }\end{array}$ & $\begin{array}{l}\text { OBSERVACIONY } \\
\text { REGISTRO }\end{array}$ \\
\hline 3 & $\begin{array}{c}\text { VISIBLE } \\
\text { SUPERFICIALMENTE } \\
\text { PERO ALTERADO } \\
\end{array}$ & $\begin{array}{l}\text { BASURALES CON FRAGMENTOS } \\
\text { FORMANDO SUELO ARQUEOLOGGICO }\end{array}$ & $\begin{array}{l}\text { OBSERVACIONY } \\
\text { REGISTRO }\end{array}$ \\
\hline 4 & ESCALA URBANA & $\begin{array}{c}\text { BASURALES CON GRAN AREA DE } \\
\text { DEPOSICIÓN }\end{array}$ & $\begin{array}{l}\text { OBSERVACION, REGISTRO } \\
\text { Y RECOLECCION } \\
\text { SUPERFICIAL }\end{array}$ \\
\hline 5 & $\begin{array}{l}\text { DECUBIERTOS POR } \\
\text { OBRAS }\end{array}$ & $\begin{array}{c}\text { BASURALES QUE SE HACEN EVIDENTES } \\
\text { DURANTE EXCAVACIONES NO } \\
\text { ARQUEOLÓGICAS }\end{array}$ & $\begin{array}{l}\text { OBSERVACION, REGISTRO } \\
\text { Y RECOLECCION } \\
\text { SUPERFICIAL }\end{array}$ \\
\hline 6 & $\begin{array}{l}\text { DEMOLICIONES Y } \\
\text { RUINAS }\end{array}$ & $\begin{array}{l}\text { FRAGMENTOS DE ESTRUCTURAS MURARIAS } \\
\text { QUE REFIEREN A POTENCIALES BASURALES } \\
\text { DOMESTICOS. }\end{array}$ & $\begin{array}{l}\text { OBSERVACION Y } \\
\text { REGISTRO }\end{array}$ \\
\hline
\end{tabular}

Figura 3- Tabla de tipologías de sitios rosarinos (basurales) en función de cómo aparecen en superficie y la prospección efectuada, adecuada a la Ley Nacional 25.743, según cada basural.

Por otro lado, lo que definió el sitio, en realidad, no fue la prospección en sí, sino el carácter estructural de una deposición o sea el contexto en el cual se hallaron los fragmentos y que sólo el profesional podría detectar en base a sus conocimientos.

Por lo tanto, la metodología empleada para este relevamiento, lejos de eludir la ley, trató de localizar los sitios de modo de no alterarlos y a la vez, de definirlos.

Así, la prospección ha dependido del sitio prospectado y no de una concepción abstracta, ya que no todo sitio fue visualmente localizable y tampoco para todos los sitios es necesaria implicaría una prospección con excavaciones o incluso retiro de materiales arqueológicos. 
Teoría y Práctica de la Arqueología Histórica Latinoamericana | Año VIII. Vol. 9 | 2019

ISSN en línea: 2591-2801 | ISSN versión impresa: 2250-866X

\section{Hacia una metodología prospectiva}

La metodología empleada en el relevamiento intentó definir la ubicación y potencialidad arqueológica de ciertos espacios urbanos en un marco histórico.

La evolución de la ciudad a partir de 1870-80 consistió en la extensión de la cuadrícula central sobre el hinterland inmediato, de modo que las calles se extendieran en el plano urbano, aunque materialmente la cuadrícula no existiera. En forma casi antagónica, formaciones pre-urbanas y sobre todo el ferrocarril preexistente dejó intersecciones con la trama urbana que resultaron inhabitables o inútiles para el trazado ferroviario, por lo que se aprovecharon para el volcado de basuras (Fernetti, 2015, p.63).

Metodológicamente, se inició una lectura histórica de esas intersecciones y su proximidad con lugares de habitación (barrios, conventillos, asentamientos) dio por resultado los sitios con basurales potencialmente antiguos, con y sin fragmentos visibles.

Los basurales se localizaron observando primeramente la documentación histórica, en especial planos urbanísticos, de detalle y de proyecto. Entre este material puede mencionarse los planos censales y varios planos de proyecto como el plano del ing. Horacio Thedy de 1903, por das ejemplos muy conocidos.

Este material gráfico -si bien escaso- permitió establecer el contacto entre los predios privados regulares (el “damero”) y los terrenos ferroviarios, la fragmentación de las lonjas originales y en general, los lugares potencialmente usados para la deposición de basuras.

Otro tipo de documentación, como la crónica periodística aunque también escasa, resultó más rica en detalles pero sesgada por la intencionalidad propia de la publicación.

Las fotografías históricas también resultaron particularmente útiles, en ese marco de escasez. Las fotografías, por lo general, también fueron intencionadas y sobre todo en los encuadres el fotógrafo evita ciertos paisajes y lugares que no deseaba mostrar. Finalmente, las descripciones realizadas en censos y otras publicaciones permitieron establecer cantidad y tipo de población, el modo de residencia y su localización.

A pesar de esta fragmentación documental, el conjunto de material histórico resultó sumamente útil para la localización de los basurales.

Inicialmente, el material permitió recorrer los espacios urbanos en forma objetivada. Los potenciales basurales se definieron por la localización cercana a poblaciones más o menos densas, como barrios o viviendas colectivas (conventillos), combinándolas con los espacios residuales o de intersección con los predios ferroviarios (paredones, plazoletas, expropiaciones, “triángulos”, “huecos” o "barrilones”).

Para el primer grupo (Tipo 1- Basurales no visibles) las prospecciones -solamente en base a la documentación- dieron por resultado unos pocos espacios pequeños, vinculados sobre todo con unidades de vivienda colectiva. En algunos casos se intentó localizar el posible espacio de deposición mediante recorridos mínimos de la basura.

Para el segundo grupo (Tipo 2- Basurales visibles superficialmente formando suelo arqueológico) y el tercer grupo (Tipo 3- Basurales visibles en superficie y con alteración en profundidad), las prospecciones visuales combinadas con la documentación localizaron y definieron la mayor cantidad de basurales.

Éstos se unificaron en un área prospectada por completo (área industrial-ferroviaria) que estuvo definida por su evolución histórica rosarina. Mediante el método de prospección visual y el agrupamiento conceptual de sitios, se elevó el área a categoría de sitio-macro con sub-sitios puntuales.

Como segunda instancia, se procedió a una observación de los suelos potencialmente arqueológicos en profundidad y basurales técnicos mediante la observación de la arquitectura remanente. 
Teoría y Práctica de la Arqueología Histórica Latinoamericana | Año VIII. Vol. 9 | 2019

ISSN en línea: 2591-2801 | ISSN versión impresa: 2250-866X

La lógica del contexto de deposición, de este modo, dependió del contexto arqueológico, o sea que fue el área general la que tuvo potencial arqueológico y no sólo el sitio localizado.

La secuencia prospectiva en el método para basurales tipos 1, 2 y 3, sería entonces enmarcar las investigaciones en la historia de Rosario, luego en la historia de las áreas históricamente formadas y posteriormente, en la definición de los espacios arqueológicos puntuales o sub-sitios.

Obviamente, es imposible una prospección general de todos los sitios potencialmente arqueológicos de Rosario mediante trincheras o calicatas exploratorias, pero es por completo viable vincular la ciudad o site-city (Cressey y Stephens, 1982, p.50) con sus sectores históricos, los cuales han generado a su vez sitios particularizados para deposición de basuras. La secuencia "de mayor a menor" como metodología implica descentrar la investigación del contexto edáfico concentrándose en la comprensión y explicación de la evolución socioeconómica de la ciudad a partir de sus paisajes culturales (Acuto, 2013, p.47), que incluyen lo arqueológico como relicto de ellos, sin por ello tener que excavar.

La solicitud de concesión de área, con esta metodología, es profesionalmente mucho más viable y requiere de menos recursos para su investigación, ya que gran parte de ésta fue efectuada durante la prospección visual.

Para el Tipo 4 (Basurales de escala urbana) la definición está dada por el cuerpo documental, dada su importancia urbana o bien por su descripción periodística, criticando el estado del lugar desde la perspectiva higienista de fines del siglo XIX. La impronta espacial de estos basurales es inmediata, con presencia de grandes volúmenes de material arqueológico, estratos confusos y extensos, quema de materiales, poblaciones aledañas, etcétera. A este respecto, el basural se comporta con la conocida dificultad del conchero prehispánico, donde las capas se borran entremezclan y los mismos materiales presentan desgastes y degradaciones propias del mismo contexto edáfico, incluso con evidencias de reuso.

En el Tipo 5 (Basurales descubiertos por obras privadas o del estado) la prospección resultó “obligada”, ya que las obras pusieron al descubierto basurales, estructuras murarias y otros elementos. De todos modos esto se redujo a un "monitoreo" en base a los permisos de construcción privados, cuyos titulares accedieron al ingreso y registro, sin tiempo a institucionalizar de algún modo la prospección.

Para el Tipo 6 (Demoliciones y Ruinas) se relevó con el mismo criterio del grupo de basurales visibles, ya que las demoliciones suelen aparecer en superficie por desgaste del manto vegetal al ser más delgada la capa humífera sobre la cabeza del muro y erosionarse. En el caso de tránsito, remociones de tierra o tareas de parquización, los restos de muro afloran y se hacen visibles. La prospección consistió en registrarlos como una categoría aparte, indicadora de un posible basural doméstico o cercanía de uno colectivo cercano. La georreferencia de las estructuras murarias, unida a otras localizaciones, podría definir espacios colectivos de arrojamiento de basuras.

\section{Los sitios arqueológicos SIH}

Ciertos sitios pudieron ser adjudicados específicamente a datos históricos precisos, datos que puntualizan una acción humana concreta, características sociales, nacionales o étnicas de las poblaciones, cronología y actividades principales o conexas.

Se definieron como sitios SIH (Sitios con Identificación Histórica) a las potenciales concentraciones de material arqueológico, visibles o invisibles, que puedan ser vinculados a datos históricos originales confeccionados en el pasado.

La noción de SIH implicó entonces, para el relevamiento, la combinación de dos tipos de fragmentos históricos: el documento y el registro arqueológico en contexto edáfico (Traba y Zuccarelli, 2014, p. 131). 
Los sitios SIH presentaron la particularidad de ser ubicados por esos datos y por lo tanto, ya presentan un "esbozo" de historia propia de la cual podrían partir las investigaciones a futuro. En total se identificaron 19 SIH con documentación histórica original.

Estos sitios pudieron estar ocultos o muy poco visibles (Tipo 1 y 5) o completamente visibles (Tipos 2, 3, 4 y 6) y su georreferencia se realizó de modo aproximado, sustentada por el carácter de su contexto de deposición posible, en espacios libres o de propietario ausente, como se vio más arriba.

Cuantitativamente, los sitios SIH resultaron casi todos visibles, aunque no puede pensarse que esto es directamente un reflejo de su importancia histórica ya que la dinámica del suelo puso afectarlos de forma tal que hayan desaparecido en gran parte.

Algunos sitios no se catalogaron como SIH aunque el material visible pudiera significar una adjudicación a cierta población. Esta referencia -supuestamente directa- no necesariamente implicaría una identificación. Por ejemplo, si bien los basurales técnicos definirían un tipo de actividad que se realizó en el pasado y de la cual fueron resultado, sería imposible, sólo en base a la observación de lo material, definir los aspectos sociales, culturales, étnicos, etcétera de la población productora original (Figura 4).

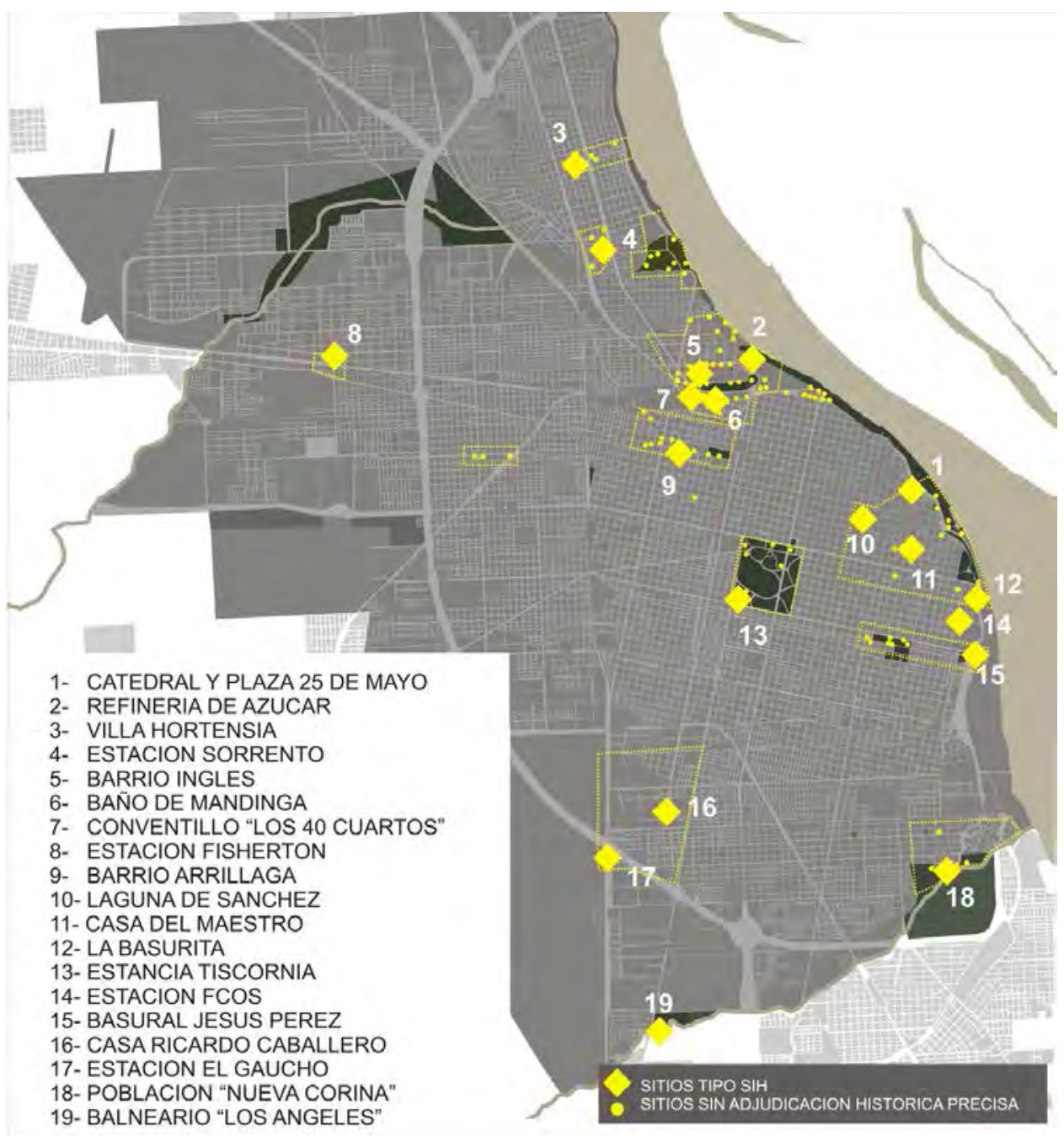

Figura 4. Plano de Sitios SIH (Sitios con Identificación Histórica) de Rosario 
Teoría y Práctica de la Arqueología Histórica Latinoamericana | Año VIII. Vol. 9 | 2019

ISSN en línea: 2591-2801 | ISSN versión impresa: 2250-866X

Podría pensarse que los sitios SIH presentan también cierto impacto en la historia rosarina, puesto que las crónicas periodísticas, fotografías o relatos orales indicarían una importancia de las poblaciones, consideradas en su momento como hechos sociales significativos. Esta consideración de la época, sin embargo, no es condicionante para que el sitio refleje esa realidad histórica, ya que los restos hallados en el sitio podrían incluso contradecir dicha importancia o cualidad y no sólo cuantitativamente. Los sitios SIH también podrían contradecir la imagen que una población actual podría tener sobre la población histórica residente y adjudicada al sitio, y el interés de la investigación -por ejemplo- podría radicar en esa divergencia.

Además, los sitios SIH tienen la cualidad de adjudicar potencialidad arqueológica a toda el área donde se encuentran inscriptos. Si un sitio SIH se consideró originalmente importante, esta relevancia históricamente definida podría ser extendida a otros sub sitios referidos a poblaciones cercanas.

\section{Los basurales intersticiales}

Si bien los sitios SIH resultaron “cómodos” de localizar, de mayor complejidad resultaron los pequeños espacios de vertido de basuras, ya que los que no han desaparecido y resultan visibles, con frecuencia no poseen documentación histórica de su momento o bien esos espacios de deposición resultaron de una costumbre irrelevante, insuficiente para ser registrados por algún medio.

En ausencia de fragmentos visibles o de documentos, el método de la historia oral resultó un buen acercamiento a la definición del basural como paisaje histórico. (Rocchietti et alt. 2008, p. 25; Rocchietti et alt., 2015, p. 248). Sin embargo, podría decirse que nunca la palabra de los vecinos definió un sitio $\mathrm{SIH}$, ya que no es "histórica" sino "historizante": el discurso de los vecinos -para los casos prospectadosnarra por lo general actividades cercanas al espacio de deposición de basuras, pero no específicamente el lugar del basural. Si bien no se avanzó sobre esta condición de tipo sociológico -considerar socialmente invisible el lugar de deposición de desperdicios- si dio numerosos indicios de la costumbre histórica en tanto no se modificaron las condiciones espaciales del basural y sobre todo la condición urbanística e inmobiliaria del predio (Fernetti, 2015, p.66 ; Fernetti, 2016, p.143).

De este modo, los espacios contra los paredones ferroviarios persistieron aún hasta hoy, formando lo que los vecinos con frecuencia llaman "tierra de nadie" o mencionan como un espacio para la "mala costumbre” de arrojar desperdicios a la vía pública. Estos indicios orales permitieron localizar algunos basurales, pero sobre todo dotar de historicidad a algunos basurales aún en uso, con estratos de basura mucho más antiguos que el visible.

En particular resultaron paradigmáticos dos sitios. Uno de ellos se denominó CEM y resultó en un basural técnico donde se arrojaron cientos de aisladores telegráficos. El espacio -casi invisible- se estableció por el testimonio de ferroviarios que sabían del lugar de una pequeña usina y una oficina de comunicaciones. El sitio PICH, en el barrio hoy denominado "Pichincha” (ex estación Sunchales, Rosario Norte) fue localizado por el testimonio del hijo de un mozo que atendía en un coche comedor ferroviario. Su relato permitió localizar un sitio donde se depositaba la basura del servicio de comedor de los trenes.

Los basurales medianos y pequeños, de menos de 50 fragmentos visibles en contexto edáfico, sólo pudieron ser detectados por la observación, una vez definido un sitio potencialmente adecuado para que se arroje la basura (Figura 5). Así, muchos pudieron ser identificados mediante la simple observación en recorridas a lo largo de las vías, veredas contra muros corridos y observación detallada de espacios públicos, sabiendo que estos espacios podían ser basurales históricos, sólo con la condición de haberse 
establecido una población en las proximidades. Dado que Rosario entre 1870 y 1920 era una ciudad "dispersa” con núcleos urbanísticos separados, el conocimiento de la historia de Rosario y la evolución de la ciudad resultó imprescindible (Volpe, 1994a, p.2).

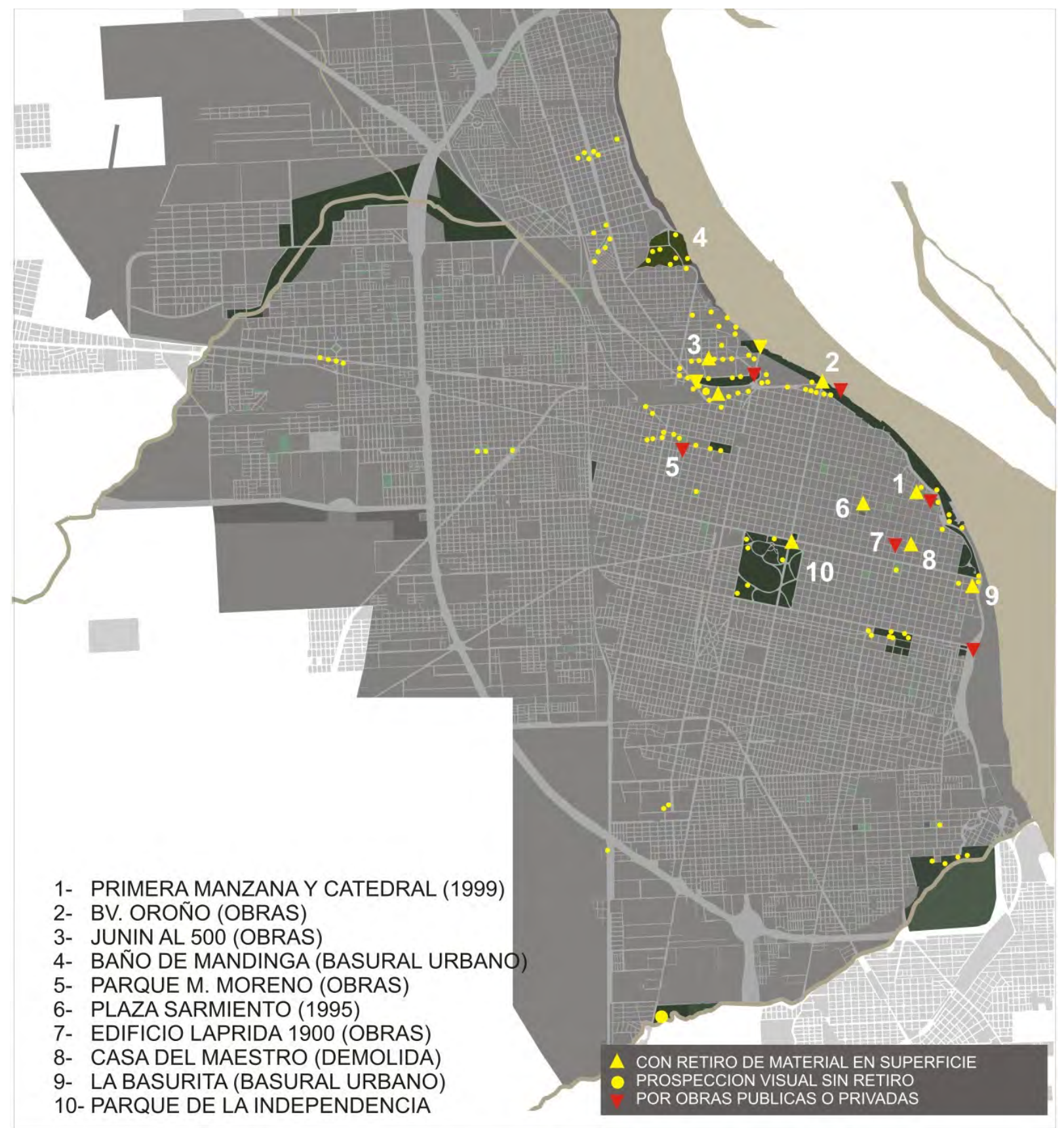

Figura 5- Plano de sitios con definición visual y recolección superficial 


\section{Conclusión: los basurales y el concepto operativo de “área arqueológica”}

El conocimiento de la historia rosarina y sus espacios sociales complejos permitieron estableces áreas dentro de las cuales ese establecieron los basurales. Inversamente, el hallazgo de basurales dentro de espacios urbanos más o menos contiguos permitió agruparlos geográficamente como un espacio que los contuviera, y al vincularlos con realidades sociohistóricas (fundaciones de barrios, poblaciones, conventillos, asentamientos). Por lo tanto, la formación de áreas arqueológicas definidas fue resultado, a la vez, empírico (se basa en lo material) y abstracto (se define en el plano) pero con un propósito pragmático: establecer la posibilidad de áreas para proyectos de investigación y por lo tanto concesionables según la Ley Nacional 25.743.

El trabajo prospectivo, de este modo, generó 127 sitios contenidos en 16 áreas específicas, cada una susceptible de generar un potencial proyecto de investigación (Figura 6).

El área -como concepto- también posibilitó una operatividad en el análisis arqueológico, ya que permite una vinculación entre los diferentes sitios que la componen. Así, los sitios formarían potencialmente una "red" que puede vincularse con las relaciones sociales, económicas y urbanísticas que formaron el espacio original, hoy representado por el área.

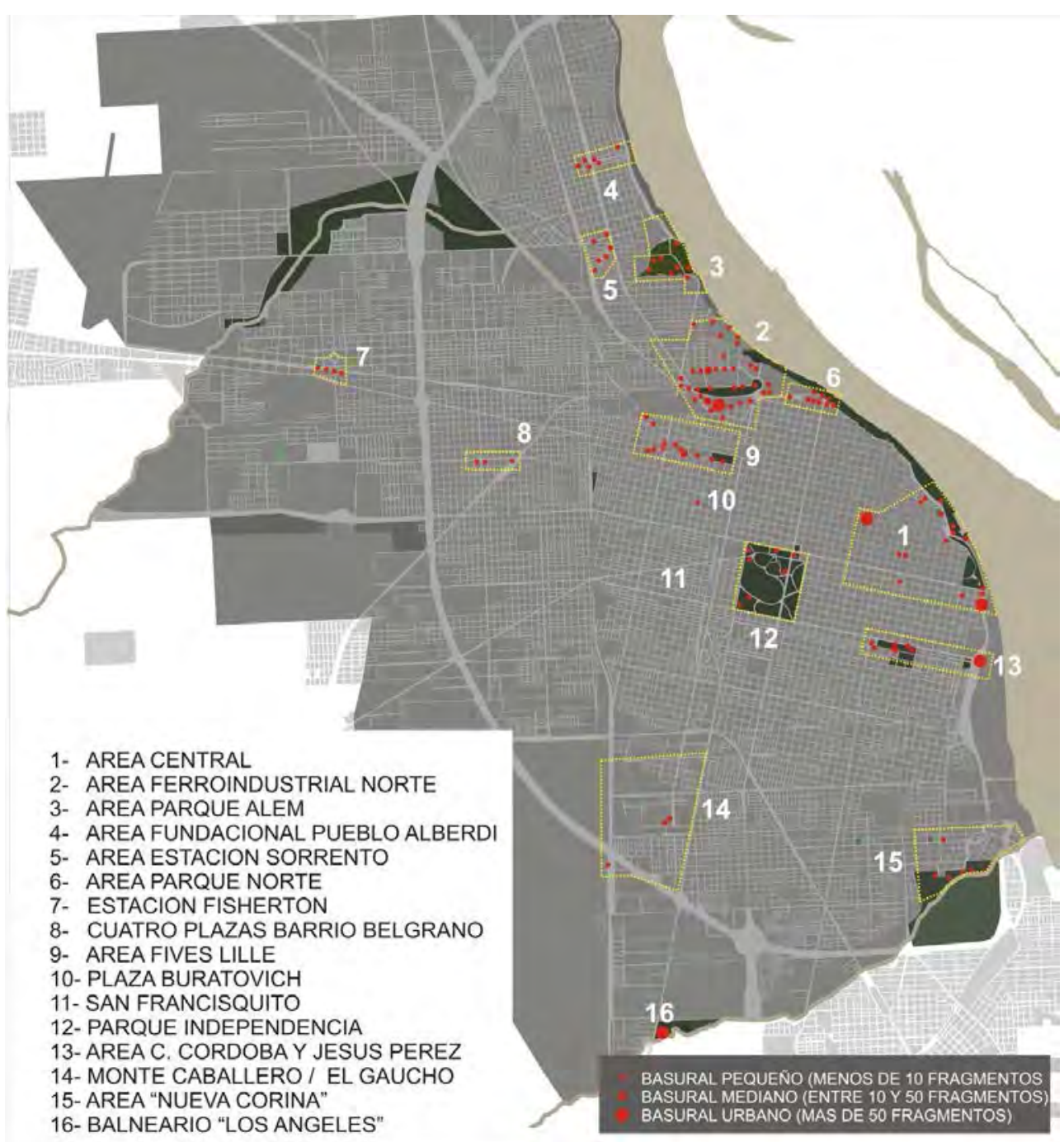

Figura 6- Plano con los 127 sitios definidos $y$ agrupados en 16 áreas históricas especificas 
Otras derivaciones son la observación cuantitativa, que indicarían la potencialidad arqueológica del área. Por ejemplo, el Área Central y el Área Ferroindustrial Norte presentaron mayor cantidad de sitios SIH y basurales Tipo 4, por lo cual podría decirse que fueron las áreas con mayor riqueza material y cantidad de documentación disponible. Varios de estos basurales ya fueron prospectados entre 1993 y 2002, justamente por su importancia.

Evidentemente, dentro de la evolución de la ciudad, éstas fueron las áreas de mayor importancia sociohistórica de Rosario.

Consecuentemente a estas consideraciones, esta mecánica de relevamiento resultó útil a los efectos de la localización y definición de la potencia arqueológica del basural y por ende, la posterior formulación del proyecto a aprobar según la Ley 25.743.

Para 2017, se comenzó con un área específica, el “Area Ferroindustrial Norte - Los Barrios Obreros”, consistente en el análisis arqueológico de un espacio urbanístico generado por la conjunción de la actividad industrial y ferroviaria entre 1870 y 1920 y concesionada por resolución N526/2017 del Ministerio de Cultura e Innovación de la Provincia de Santa Fe. Como demostración de potencialidad del Area Ferroindustrial Norte concesionada, ésta ha generado, 35 sitios prospectados incluyendo 2 basurales técnicos, con 11 sitios analizados al momento.

El área posee por lo tanto una gran riqueza del registro arqueológico (más de 1000 fragmentos) en un contexto de gran dinámica edáfica. Puede pensarse que otras áreas poseen una riqueza similar. También la agrupación por áreas de origen sociohistórico además de índole arqueológica, permitiría metodologías comparativas entre áreas y sitios.

Como conclusión, podría argumentarse que la prospección per se y la sola identificación de sitios, debe vincularse y articularse con una lógica sociohistórica además de arqueológica. De este modo, el relevamiento dejaría de ser un registro de contextos de deposición para convertirse un registro de contextos arqueológicos más complejos y reveladores y por lo tanto, potencialmente útiles para el conocimiento de la historia de Rosario.

\section{Notas}

${ }^{1}$ Se empleó este concepto dado que es una manera de entender la ciudad como un todo arqueológico, en tanto el desarrollo de la misma tuvo un único factor económico unificador, la introducción del capitalismo. Así, en contexto edáfico pueden hallarse diferentes materiales, pero cuyo origen es el mismo, el modo de producción capitalista. La ciudad, desde lo arqueológico, sería un gran sitio en el cual hay desagregaciones según la composición social o la actividad económica, pero con un factor común que define éstas.

${ }^{2}$ La ley fue recuperada del sitio web: http:/www.conicet.gov.ar/wp-content/uploads/Ley-N\%C2\%BA25743-Protecci\%C3\%B3n-del-Patrimonio-Arqueol\%C3\%B3gico-y-Paleontol\%C3\%B3gico.pdf

\section{Referencias bibliográficas}

ACUTO, F. (2013). ¿Demasiados Paisajes? Múltiples teorías o múltiples subjetividades. En: Anuario de Arqueología No5. Rosario: Departamento de Arqueología. Escuela de Antropología. Pp. 31-50.

ARMUS, D. y HARDOY, J. (2014). Vivienda popular y crecimiento urbano en el Rosario del novecientos. En: Revista EURE - Revista De Estudios Urbano Regionales No31. Santiago de Chile. 
Teoría y Práctica de la Arqueología Histórica Latinoamericana | Año VIII. Vol. 9 | 2019

ISSN en línea: 2591-2801 | ISSN versión impresa: 2250-866X

COLASURDO, M. B. y SARTORI, J. I. (2011). La conformación de la etnicidad a partir de los hábitos alimenticios: su abordaje desde la antropología y la arqueología histórica. En: Revista de Arqueología Histórica Argentina y Latinoamericana 5. Buenos Aires. Pp. 125-146.

COLASURDO, M. B. (2012). Análisis del registro arqueológico de dos basureros del siglo XIX de la ciudad de Rosario: primeras aproximaciones. En: Anuario de Arqueología nº 4. Pp. 269-281.

CRESSEY, P. y STEPHENS. J. (1982). The City-Site Approach to Urban Archaeology. En R. Dickens Jr. (editor), Archaeology of Urban America. The Search for Pattern and Process,. Nueva York: Academic Press,. Pp. 41-59.

FERNETTI, G. (2015). Relevamiento y potencial arqueológico de antiguos basurales en los barrios Refinería y Talleres de Rosario. En: Revista Teoría y Práctica de la Arqueología Latinoamericana. Año IV Vol. 4. Verano 2015. Buenos Aires: Centro de Estudios de Arqueología Histórica. UNR. Aspha Ediciones: Pp. 59-68.

FERNETTI G. (2016). ¿Qué era el “barrio inglés”? Arqueología de un conjunto de viviendas del Ferrocarril Central Argentino, Rosario, Argentina. En: Revista Teoría y Práctica de la Arqueología Latinoamericana. Año V. Vol. 5. Verano 2015. Buenos Aires: Centro de Estudios de Arqueología Histórica. UNR. Aspha Ediciones:. Pp. 145-158.

GALIMBERTI, C. (2016). Planificar el territorio metropolitano: Historia de la planificación regional de Rosario (1935-1976). En Mendoza: Revista Historia Americana. Vol.51 no.1: Pp.

FALCÓN, R. (2005). La Barcelona argentina. Migrantes, obreros y militantes en Rosario, 1870-1912, Rosario: Laborde Editor.

LÓPEZ LILLO, J. (2013). Problemas de la vida cotidiana: algunas reflexiones teóricas para un análisis social en Arqueología de la domesticidad. En: Gutiérrez Lloret y Grau Mira, eds. De la estructura doméstica al espacio social. Lecturas arqueológicas del uso social del espacio. Alicante: Publicaciones de la Universidad de Alicante. Pp. 330-348.

MUGICA M. L. y MARTIN, M. P. (2001). La sociedad rosarina en el siglo XX: cambio, vida cotidiana y prácticas sociales. En Falcón, R y M. Stanley La Historia de Rosario. Tomo I: 157 - 226.

ROCCHIETTI, A; SIMONASSI, S. y GERGOLET, S. (2008). Curtiembre Noguera: arqueología y barriadas obreras. En: IX Congreso Argentino de Antropología Social. Misiones: Facultad de Humanidades y Ciencias Sociales. UNM, Posadas. Pp. 12-28.

ROCCHIETTI, A., DE GRANDIS, N. y VALENTÍNI, M. (2015). Rosario de Santa Fe y su paisaje cultural: el basural de Jesús Pérez, La Tablada. En: III Jornadas Binacionales de Paisajes Culturales en Patagonia Argentina y Chile, Comodoro Rivadavia. Pp. 243-250.

SCHIFFER, M. (1990). Contexto arqueológico y contexto sistémico. En: Boletín de Antropología Americana 22. México: Instituto Panamericano de Antropología e Historia. Pp.80-93.

TRABA, D. y ZUCCARELLI, V. (2014). Arqueología y fuentes históricas. Diálogos interdisciplinarios. En: Revista Diálogos. vol. 4, No. 2. Julio 2014. Pp. 121-138 San Luis: UNSL - Facultad de Ciencias Humanas: Pp. 121-138. 
VOLPE, S. (1994a). Excavaciones en la Plaza 25 de mayo. En: Informe interno de la Escuela de Museología. Municipalidad de Rosario.

VOLPE, S. (1994b). Tipología de recipientes de gres cerámico y precintos de cerveza: excavaciones arqueológicas en Rosario. En: Revista Arqueología Urbana N⒚ Centro de Arqueología Urbana. Buenos Aires: Universidad de Buenos Aires.

VOLPE, S. (1994c). Catálogo de vajillas de loza inglesa en Rosario, Argentina. Rosario: Escuela de Museología. Secretaría de Cultura, Municipalidad de Rosario.

VOLPE, S. (2000). Arqueología de salvamento. La primer manzana el Rosario temprano (1790-1820) Excavaciones en la Plazoleta Emilia Bertolé. En: I ${ }^{\circ}$ Congreso Virtual de antropología y arqueología. NAyA. Recuperado de: http://www.equiponaya.com.ar/congreso2000/ponencias/Soccorso_Palma. htm

Recibido: 10 de junio de 2019

Aceptado: 20 de octubre de 2019 